\title{
Rietveld texture analysis from synchrotron diffraction images. I. Calibration and basic analysis
}

\author{
Luca Lutterotti, ${ }^{1}$ Roman Vasin, ${ }^{2,3}$ and Hans-Rudolf Wenk ${ }^{2, a)}$ \\ ${ }^{I}$ Department of Industrial Engineering, University of Trento, Italy \\ ${ }^{2}$ Department of Earth and Planetary Science, University of California, Berkeley, California 94720 \\ ${ }^{3}$ Frank Laboratory of Neutron Physics, Joint Institute for Nuclear Research, Dubna, Russia
}

(Received 1 March 2013; accepted 17 October 2013)

\begin{abstract}
Synchrotron X-ray diffraction images are increasingly used to characterize not only structural and microstructural features of polycrystalline materials, but also crystal preferred orientation distributions. Diffraction data can be analyzed quantitatively and efficiently with the Rietveld method and here the detailed procedure is reported from the experiment to the calibration of the two-dimensional detector and full analysis of the sample. In particular, we emphasize the advantage of doing the calibration inside the Rietveld least-squares fitting instead of a preliminary graphical calibration. Then the procedure is described to quantify crystal preferred orientations with the Rietveld method implemented in software "Materials Analysis Using Diffraction". The process is illustrated for a US "nickel" coin, a 75 at.\% copper 25 at.\% nickel alloy with face-centered cubic structure and a strong cube texture. (C) 2014 International Centre for Diffraction Data.
\end{abstract}

[doi:10.1017/S0885715613001346]

Key words: diffraction images, synchrotron diffraction, Rietveld method, texture analysis

\section{INTRODUCTION}

Recently we have described a procedure to obtain quantitative texture information from time-of-flight (TOF) neutron diffraction spectra and this has been applied to several instruments such as HIPPO at the Lujan Center of Los Alamos National Laboratory (Wenk et al., 2010) and SKAT at the Joint Institute for Nuclear Research in Dubna, Russia (Wenk et al., 2012). Here, we describe a similar procedure for $\mathrm{X}$-ray diffraction images, particularly images collected at synchrotron radiation sources. These $\mathrm{X}$-ray images need to be calibrated and transformed to diffraction patterns and a correct procedure for the calibration is important to obtain optimal results. In addition, images collected in situ at high pressure may show distortions resulting from the influence of anisotropic stress. Smaller distortions are also present in the case of residual stresses and a special procedure is necessary to separate distortions because of detector errors from effects of lattice strains. The high resolution of X-ray diffraction images also allows characterization of microstructural characteristics such as grain size and grain shape.

Emphasis is on quantitative characterization of preferred orientation patterns in polycrystalline aggregates. The orientation of crystals (or texture) has a profound influence on anisotropic physical properties, which depend on the properties of single crystals and the orientation distribution. Texture has long been studied in metals and rocks [see Kocks et al. (2000) for details and applications] and we assume that the reader is familiar with the basic principles of texture analysis and texture representation. Texture or crystallographic preferred orientation $(\mathrm{CPO})$ describes the orientation of

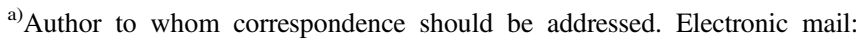
wenk@berkeley.edu
}

crystallites of phases that compose a material, relative to sample coordinates, using a three-dimensional (3D) statistical orientation distribution function (ODF). Even though the computer programs used for data analysis rely on the ODF, we will only use pole figures for representation. A pole figure is a 2D projection of the 3D ODF and is easier to visualize. It displays the probability density of finding poles perpendicular to $h \mathrm{kl}$ lattice planes relative to the sample coordinate system. Textures are not only important for assessing deformation histories and calculation of physical properties of polycrystals, they are also essential for crystal structure refinements (e.g. Lutterotti and Bortolotti, 2005; Grässlin et al., 2013), quantitative phase analysis of aggregates with preferred orientations, as well as the analysis of macroscopic stress states (e.g. Lutterotti et al., 2004). Since texture analysis is the emphasis of this presentation, we recommend the same procedure for synchrotron diffraction image analysis of samples with random crystallite orientation, including structure analysis of highly disordered materials (e.g. Battocchio et al., 2012).

Experimental methods used to obtain diffraction images are briefly described. The Rietveld method implemented in the program package Materials Analysis Using Diffraction (MAUD), which was originally developed for neutron diffraction (Lutterotti et al., 1997), was then applied to X-ray diffraction images. Since the first such application to dinosaur tendon and salmon scale (Lonardelli et al., 2005), procedures have been refined and have become standardized. A metal coin (US "nickel") is used as an example for data analysis. This sample is very straightforward to analyze; thus beginners should be able to follow our procedure on their own. In the Rietveld code MAUD, there are many options on how to proceed to arrive at the same answer. Here, we follow one (and occasionally point out other possibilities) leaving it to the more experienced user to discover the full variety. 
Readers should first consult our publication on neutron diffraction texture analysis (Wenk et al., 2010) where the situation is more straightforward and where many options are described, including graphic representation and texture displays. This will not be repeated here. In a companion paper (Part II), we will extend the method to complex materials such as multiphase shale and in situ deformation studies at ultrahigh pressure (Wenk et al., 2014). In two on-line attachments, we describe step-by-step procedures for data analysis in MAUD and provide data sets that can be used by readers.

\section{DIFFRACTION EXPERIMENTS}

In a typical synchrotron diffraction texture experiment, monochromatic radiation is used, the sample is analyzed in transmission, and the diffraction image is recorded with a CCD camera or an image plate detector (Figure 1). For the coin sample, high-energy (hard) $\mathrm{X}$-rays with a short wavelength $(\lambda=0.10798 \AA$ ) were used. Hard X-rays have high penetration depth and for a medium absorbing material the sample dimension can be up to $2 \mathrm{~mm}$ in thickness without significant intensity loss. The X-ray diffraction measurements were done at the BESSRC 11-ID-C beamline of the Advanced Photon Source (APS) at Argonne National Laboratory. Similar experiments can be done at other beamlines on different synchrotron sources (among them ALS, ESRF, HASY, etc.). The specimens are typically prepared into $1-2 \mathrm{~mm}$ thick slabs with parallel sides and mounted on a goniometer with an aluminum pin for rotation (Figure 2). The beam size is about $0.5-1 \mathrm{~mm}$ in diameter, and the detector, in the case of our experiment, was positioned at a distance of about $1850 \mathrm{~mm}$ from the sample. The angular range that is recorded depends on sample-to-detector distance.

Figure 3(b) shows a diffraction image of an American "nickel" coin, composed mainly of copper, with Debye rings corresponding to reflections on $h \mathrm{kl}$ lattice planes. The azimuthal variations of $\mathrm{X}$-ray intensity immediately indicate lattice preferred orientation. Debye rings are smooth, indicating excellent grain statistics, which is a prerequisite for a quantitative texture analysis with the Rietveld method.

As the relative orientations of crystals and sample are crucial to texture analysis, the diffraction image recorded by the detector must correspond to a view from the sample in the direction of the incident beam. This is not trivial and can be verified by taking an image with an object placed in front of

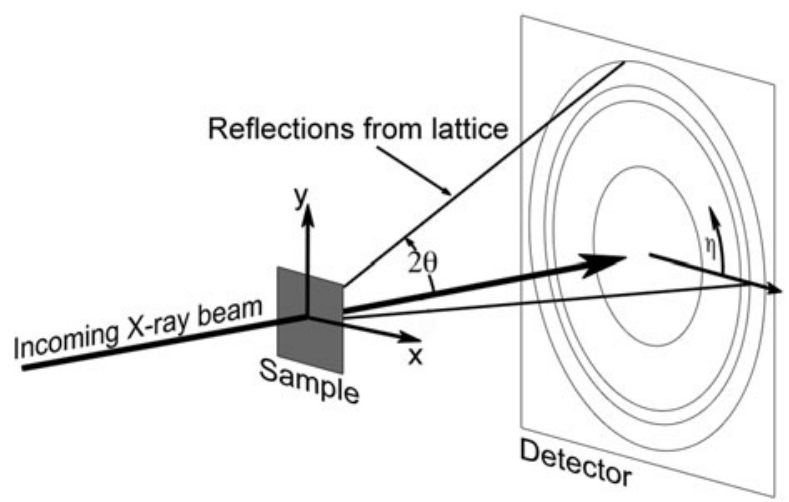

Figure 1. Geometry of a synchrotron diffraction texture experiment. Axis $x$ is the goniometer rotation axis. Azimuthal angle $\eta$ is before image rotation during MAUD processing.

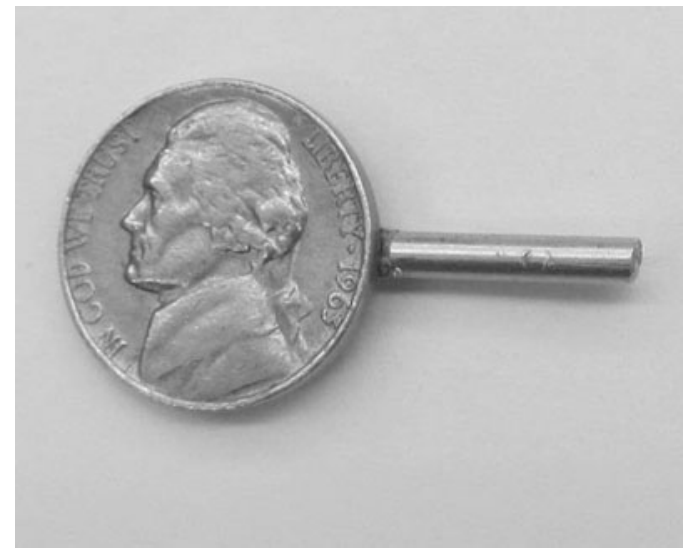

Figure 2. Typical sample for a hard X-ray diffraction experiment: nickel coin mounted on a pin.

the detector that produces an asymmetric shadow, and then viewing the image to verify its correct orientation. It may be necessary to invert or rotate the image prior to the data analysis.

A diffraction image contains reflections from many lattice $h \mathrm{kl}$ planes of many crystals, each obeying Bragg's law. In terms of pole-figure coverage on a sphere, this corresponds to two small circles [because of the center of symmetry imposed in a diffraction experiment, Figure 4(a)]. A single diffraction image is not very satisfactory for pole-figure coverage and often is not enough for quantitative texture analysis. In order to improve coverage, the sample is therefore rotated around an axis perpendicular to the incident beam $[x$ in Figure 1 and the $Y_{M^{-}}$axis in Figure 4(a)]. Generally, images at different omega angles $(\omega)$ are recorded and correspondingly the coverage is improved [Figure 4(b)]. Information from several images has to be combined for quantitative texture analysis. In the case of the nickel coin, five images were collected at $\omega=-40^{\circ},-20^{\circ}, 0^{\circ}, 20^{\circ}, 40^{\circ}$, providing a coverage as shown in Figure 4(b). To further improve coverage, the sample could be mounted in different directions and corresponding images could be combined. This was not done here. During the exposure, the sample can also be translated along the horizontal axis to increase the volume average and grain statistics (in this case from -2 to $+2 \mathrm{~mm}$ ). The sample coordinate system is defined by three axes $X_{M}, Y_{M}$, and $Z_{M}$ and corresponding rotations $\chi, \omega$, and $\varphi$ define the orientation of the sample [Figure 4(a)].

The X-ray diffraction experiment has to satisfy the following conditions:

- The wavelength must be known to refine instrument parameters.

- The precise location of the primary X-ray beam needs to be established by using a metal pin mounted on a goniometer head to assure the beam is at the center of all goniometer rotations. In our case, the large instrument goniometer needs to be translated until the horizontal $\omega$ rotation axis is in the beam center. Generally, this center position is viewed through a telescope with crosshairs for mounting samples for texture analysis in the correct position and the axis is rotated to verify that no movements of the pin tip can be detected through the telescope. 
(a)

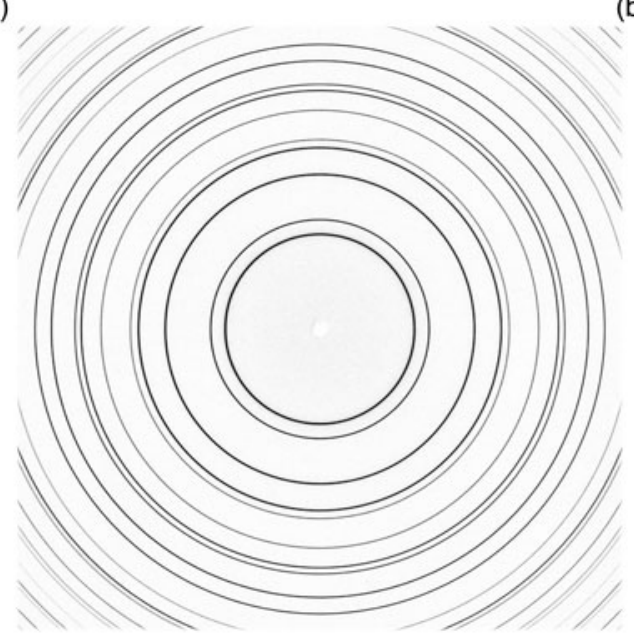

(b)

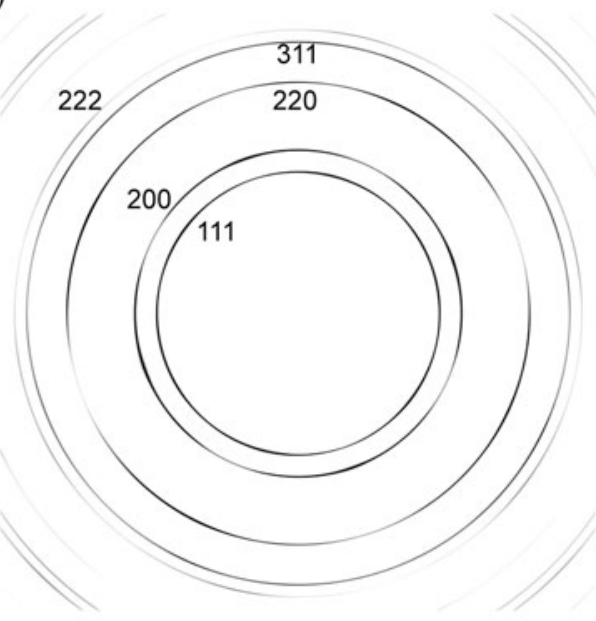

Figure 3. Diffraction images with Debye rings measured at APS 11-ID-C. (a) $\mathrm{CeO}_{2}$ standard, (b) nickel coin, $\omega=0^{\circ}$. Images are before rotation in ImageJ.

- When measuring textures, it is imperative to know the orientation of the sample relative to the diffraction instrument, and thus the directions of diffraction vectors relative to the sample for all diffraction patterns. Make necessary sketches! Figure 2 shows the coin as viewed from the incident beam. Be sure that sample tilts correspond to $\omega$-rotations as illustrated in Figure 1.

- The sample needs to be centered in the $\omega$ rotation axis to ensure the same volume element and sample-detector distance at all tilt angles. Usually this is done with a small goniometer head mounted on the large instrument goniometer. On it the sample can be translated and rotated.

- The exact detector distance and detector orientation, as well as instrument peak broadening parameters need to be calibrated at least once. This is done with a powder standard such as cubic $\mathrm{CeO}_{2}(a=5.4116 \AA$, see SRM674b-NIST certificate and also Yashima et al., 2003) or $\operatorname{LaB}_{6}(a=4.156$ $89 \AA$, see SRM660b-NIST certificate, and also Chantler et al., 2004) just before and/or immediately after the diffraction experiment on the textured sample. With $\mathrm{LaB}_{6}$ a higher $d$-range is accessible. Using the standard the detector geometry and instrumental part of diffraction peak broadening are refined for the calibration and then kept constant during the Rietveld analysis of the sample.
- You need to know detector dimensions and characteristics. As an example the Perkin Elmer amorphous silicon large area detector used for the coin study at APS has dimensions of $2048 \times 2048$ pixels and a pixel size of $200 \times 200 \mu \mathrm{m}$.

\section{INSTRUMENT AND DETECTOR CALIBRATION WITH THE RIETVELD METHOD}

While experiments are fast, data analysis is quite involved to advance from diffraction images to quantitative orientation distributions. The Rietveld method (Rietveld, 1969) was originally developed for structure analysis from neutron powder diffraction patterns and has been greatly expanded since then. Currently, freely available software versions that implement quantitative texture analysis are the General Structure Analysis System (GSAS; Von Dreele, 1997; Larson and Von Dreele, 2004) and Materials Analysis Using Diffraction (MAUD; Lutterotti et al., 1997; Lutterotti, 2010). Vogel et al. (2004) compared results for neutron diffraction texture analysis by these two programs.

In this section, we focus on image processing and calibration. Independent from the type of analysis to be done, we need to measure and analyze first an appropriate standard to calibrate the $2 \mathrm{D}$ detector (distance, center, tilting, and also (a)

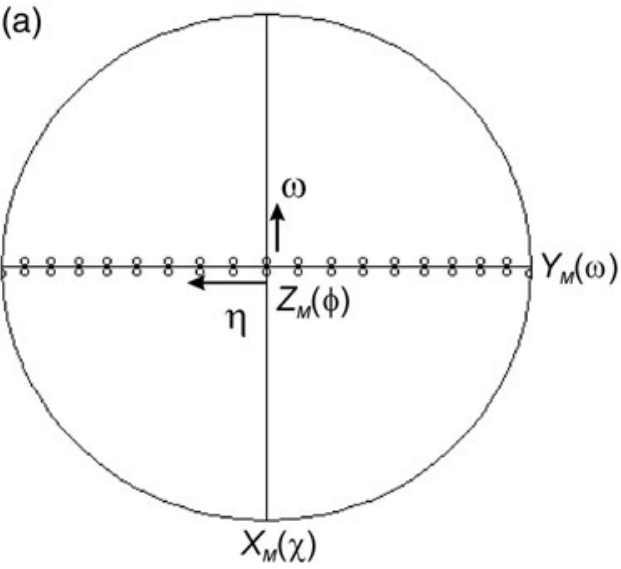

(b)

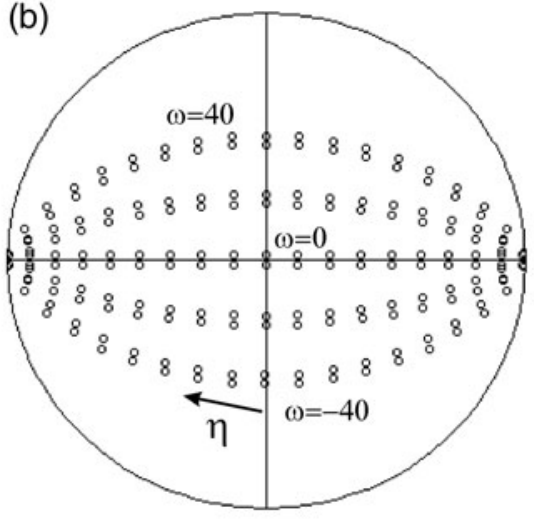

(c)

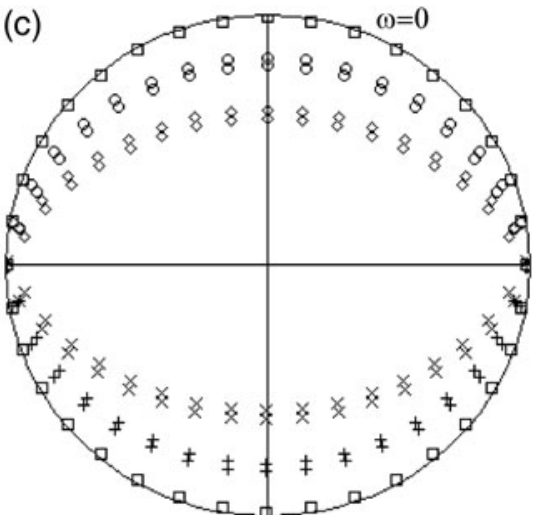

Figure 4. Pole-figure coverage with a single image (a) and with the sample rotated to different positions (b). Definition of rotation angles is indicated. (c) Coverage after a $90^{\circ}$ rotation to obtain a view looking down on the coin. ( $10^{\circ}$ is used for $\eta$ increment). 
the wavelength if it not known with accuracy) and then use the calibration for the subsequent analyses with the actual samples.

One way to perform the detector/image calibration is with graphical routines as implemented in software Fit2D (Hammersley, 1998, download from http://www.esrf.eu/computing/scientific/FIT2D/). In Fit2D the "calibrant" method is used for the standard. After choosing a few points on an inner Debye ring, the program will find the best center ( $x$ and $y$ detector coordinates), detector tilting errors and detector distance or wavelength, by fitting the maximum intensities on a certain number of points on the Debye rings with the $d$-spacings from the standard. We advise not refining both distance and wavelength as they are highly correlated at low angles (high energy). Here we did not refine the wavelength. The procedure of Fit2D is quite precise and accurate, but in this paper we propose a different way to perform the detector calibration to resolve possible problems arising later with the analysis of the actual samples, in particular:

- The Fit2D procedure treats the deviation of Debye rings from perfect circular shape (i.e., ovals) as due to tilting errors of the detector, excluding other causes such as macroscopic mechanical strains in the sample as for in situ highpressure experiments. This could be corrected if the centering of the images does not change between the standard and the strained sample, but this is often not the case.

- In the case of a highly textured sample or a nanocrystalline material, it is very difficult to determine accurately the image center graphically using these procedures as the Debye rings are more like distributed points or segments, or the lines (especially of multiphase/disordered materials) are too broad for a precise localization of the maxima.

- In case the texture is not of interest, a common procedure is to integrate over the whole Debye rings to reduce everything to only one "randomized" pattern. Eventual centering errors or deviation of rings from round shape are then causing additional broadening of the diffraction lines with no possibility to correct such errors later.

The procedure implemented in the Rietveld software MAUD (Lutterotti, 2010) relies instead on a first reduction of the diffraction image into several patterns using a graphical centering with no tilting errors. This pre-centering does not need to be perfect. The original image coordinates are recorded for each point of each pattern. The errors in centering and tilting are subsequently refined during the Rietveld refinement along with the other parameters, using a calibration function that calculates each time the correct $2 \theta$ value of all data points based on the actual values of such parameters.

The formula used for the transformation of the image coordinates into $2 \theta$ is the following:

$$
\cot (2 \theta)=\frac{D}{r \cos \delta}-\tan \delta
$$

where

$$
\begin{gathered}
\tan \delta=\frac{-x \cos \delta_{y} \sin \delta_{x}+y \sin \delta_{y}}{r} \\
r=\sqrt{x^{2}+y^{2}}
\end{gathered}
$$

$$
x=x_{\mathrm{r}} \cos \eta-\Delta x_{\mathrm{c}}
$$

is the image coordinate $x$ from the true center

$$
\begin{aligned}
& y=x_{\mathrm{r}} \sin \eta-\Delta y_{\mathrm{c}} \\
& \text { is the image coordinate } y \text { from the true center }
\end{aligned}
$$

$D$ is the sample-to-detector distance, $\delta_{x}$ is the detector tilting along the $x$-axis (rotation around the $y$-axis), $\delta_{y}$ is the detector tilting along the $y$-axis (rotation around the $x$-axis), $\Delta x_{\mathrm{c}}$ is the $x$ centering error, and $\Delta y_{\mathrm{c}}$ is the $y$ centering error.

During the reduction of patterns, we save for each point the radial distance from the original center and the angle $\eta$ of the pattern [angular coordinate around the Debye rings, from $0^{\circ}$ to $360^{\circ}$, Figure $\left.4(\mathrm{a})\right]$.

This procedure has several advantages:

- the calibration inside the Rietveld least-squares refinement is much more effective and accurate than on the image because it uses the entire peak profile and not only a maximum of the circle lines;

- the centering can be refined, also for difficult cases such as nanocrystalline sample, fiber diffraction or highly textured samples;

- we can separate the deviation of Debye rings from round shape because of anisotropic macroscopic strains from detector tilt, as these two have a different dependence on $2 \theta$;

- we generally observe an improved peak resolution and separation using this procedure over Fit2D because of the better centering and tilting correction (especially for high-angle/ low-energy experiments).

The procedure is quite efficient and can be applied also to detectors in asymmetrical positions and not perpendicular to the beam. In this paper, we will only show the case of a detector roughly perpendicular and centered with respect to the beam.

The procedure in MAUD always corrects the $2 \theta$ position of the experimental points rather than the calculated position of the diffraction peaks. The same applies to other instrumental errors like a $2 \theta$ displacement used in the traditional BraggBrentano geometry. The Fit2D and MAUD methodologies give similar results as long as the $2 \theta$ correction is small.

In Table I, we compare results of the detector calibration using the "calibrant" procedure in Fit2D and by fitting directly in Maud. Two standards and wavelengths have been used. The $\mathrm{CeO}_{2}$ corresponds to the APS experiment, $\mathrm{LaB}_{6}$ data were collected at XRD1 of the Elettra synchrotron in Trieste (image plate detector). The center $y$ coordinate in Fit2D has been converted to the MAUD convention (the 0 for $y$ is on the top-left corner and the coordinate axis points down; in Fit2D is lowerleft corner and points up). Also the signs of the detector tilts have been reversed in Fit2D to compare with the MAUD convention. We notice considerable differences, far beyond the estimated standard deviation.

The stack of experimental $\mathrm{CeO}_{2}$ patterns can be viewed with the "Plot 2D" tab in which all individual 72 patterns are displayed [Figure 5(a)]. This image is after instrument corrections have been refined and diffractions appear as straight lines. Figure 5(b) is the sum of individual patterns ("Plot") with tick marks below showing positions of diffraction peaks. Crosses are experimental data points and the line 
TABLE I. Comparison of the detector calibration results for $\mathrm{CeO}_{2}$ and $\mathrm{LaB}_{6}$ using the "calibrant" procedure in Fit2D and then fitting in MAUD or by fitting directly in MAUD the calibration parameters of the detector. Parameters without standard deviations (in parentheses) have not been refined in MAUD.

\begin{tabular}{|c|c|c|c|c|}
\hline \multirow{2}{*}{$\begin{array}{l}\text { Standard }(\lambda) \\
\text { Calibration }\end{array}$} & \multicolumn{2}{|c|}{$\mathrm{CeO}_{2}(0.10798 \AA)$} & \multicolumn{2}{|c|}{$\mathrm{LaB}_{6}(0.7093 \AA)$} \\
\hline & Fit2D & MAUD & Fit2D & MAUD \\
\hline Center $x(\mathrm{~mm})$ & 205.189 & $205.0897(3)$ & 81.772 & $81.745(2)$ \\
\hline Center $y(\mathrm{~mm})$ & 204.751 & $204.7559(4)$ & 81.674 & $81.6395(2)$ \\
\hline$\delta_{x}\left({ }^{\circ}\right)$ & -0.093 & $-0.080(3)$ & 0.258 & $0.2899(6)$ \\
\hline$\delta_{y}\left(^{\circ}\right)$ & 0.092 & $0.031(4)$ & -0.591 & $-0.6417(6)$ \\
\hline Distance (mm) & 1852.085 & $1850.584(2)$ & 129.3505 & $129.344(3)$ \\
\hline$a$ (calibration) & 5.4116 & 5.4116 & 4.15689 & 4.15689 \\
\hline$a$ (refined, MAUD) & $5.41312(3)$ & 5.4116 & $4.15707(3)$ & 4.15689 \\
\hline$R_{\mathrm{wp}}(\%$, MAUD $)$ & 4.14 & 8.4 & 2.13 & 6.91 \\
\hline $2 \theta$ error (MAUD) & $-0.00088(3)$ & - & $-0.001(2)$ & - \\
\hline
\end{tabular}

represents the Rietveld fit. Note that it reproduces not only positions and intensities but also peak shapes. For diffraction data, a square root scale for intensity is quite useful, as smaller peaks are more visible.

The full step-by-step procedure in MAUD to calibrate the detector and instrument parameters is reported in Appendix 1 (available online at http://www.journals.cambridge.org/PDJ) and corresponding data files can be downloaded from http:// PD-journal.

\section{NICKEL COIN ANALYSIS}

Once we have calibrated the detector and determined the instrument resolution we can proceed with the analysis of the coin.

To perform a texture analysis we need a sufficient number of data in order to measure a representative number of grains in different orientations. We need to cover the entire orientation space to ensure the validity of the results. For the estimation of the microstructure, we rely on diffraction line broadening and for this it is critical to have a good characterization of the instrumental line broadening that is obtained during the experiment with the standard. The refinement strategy for the microstructural characteristics of the sample in MAUD is different than in other Rietveld programs such as GSAS. There is a clear separation between the instrument line broadening model and the line broadening model related with the sample. This permits to refine directly only the quantities of interest concerning the sample and keep constant parameters associated with the instrument.

The general procedure with the sample analysis is to do the integration of each image in slices using the same center and parameters used for the integration of the standard image. Then we use the same distance, centering and tilting errors as refined in the standard analysis. If the beam centering did not change we should have a perfect calibration of the patterns (i.e., transformation of detector coordinates to $2 \theta$ angles). But having all the patterns around the center separated in slices, we can also refine centering errors if necessary. In this analysis, we will refine the centering for the sample, as in general it is not the same as for the standard. The center of the Debye rings corresponds to the position of the diffracting volume of the sample. For the coin sample it correspond to the beam position as the beam is entirely inside the sample; but the $\mathrm{CeO}_{2}$ standard powder was in a capillary and thus the standard was entirely in the beam, and its position inside the beam defined the centering.

The only difference between the image integration for the standard is that for the sample we choose a different azimuthal integration step around $\eta$ (eta) angle based on the texture strength and/or grain statistics. We use a $5^{\circ}$ step to integrate along the Debye rings (corresponding to 72 patterns per image). It is important to check the original diffraction image and regular intensity variations around Debye diffraction rings. The size of the integration step should be chosen
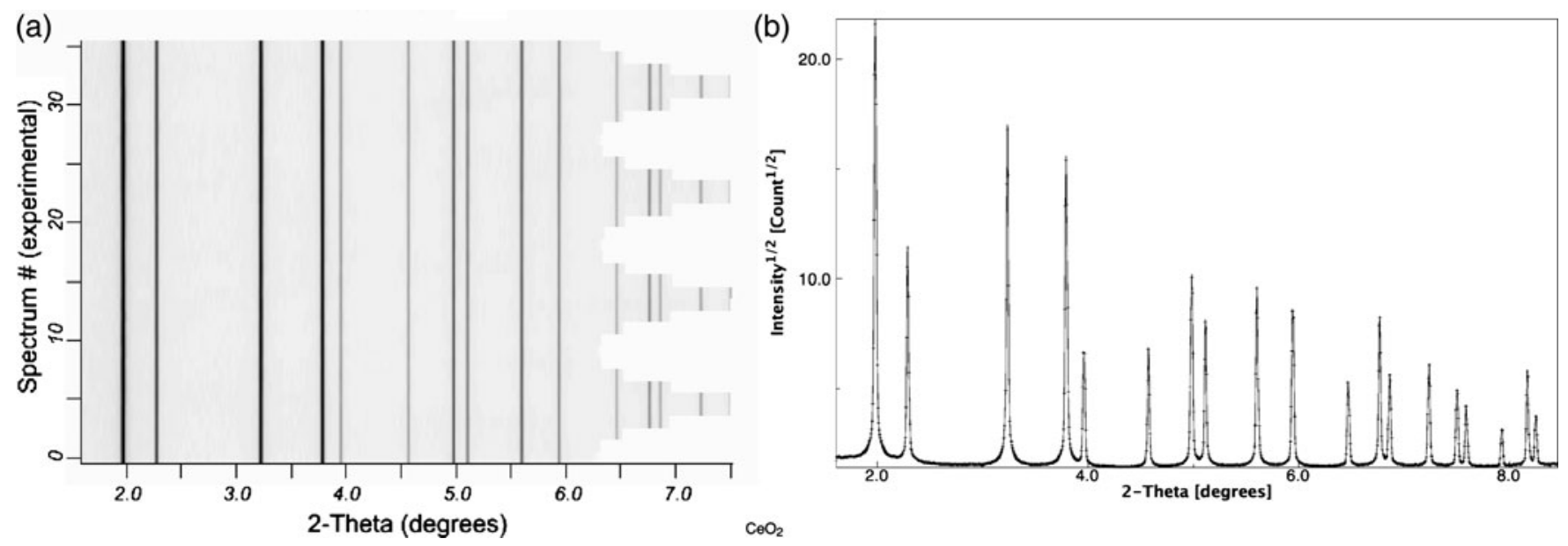

Figure 5. Diffraction patterns for $\mathrm{CeO}_{2}$. (a) Stack of experimental diffraction patterns after refinement of detector parameters; diffraction lines are straight (b). Fit of all 36 patterns of (a) summed together using the calibration procedure inside MAUD. 
sufficiently small, so that variations (and corresponding ODF peculiarities) are clearly distinguishable. However, intensity variations due to poor grain statistics ("spotty" diffraction images) require larger integration steps to effectively increase grain statistics. We have to determine if one image is sufficient to calculate the ODF. It depends on the crystal symmetry of the compound, on sample symmetry and, in principle, on the orientation distribution itself (e.g., when dealing with a polycrystalline phase where large parts of orientation space have corresponding ODF values equal to zero, the amount of necessary measurements greatly decreases, but such information is rarely available beforehand). In general, it is advantageous to have several images providing a wider pole-figure coverage [Figures 4(b) vs. 4(a)].

We will start the analysis using only one image $\left(\omega=0^{\circ}\right.$, with the beam perpendicular to the coin's face) [Figure 4(a)] and then add other images to improve the ODF coverage [Figure 4(b)]. In the tutorial (Appendix 2, available online at http://www.journals.cambridge.org/PDJ), we will show the step-by-step procedure with all images. The image has been loaded as for the standard and integrated in $5^{\circ}$ steps in $\eta$ to transform it into 72 diffraction patterns (note that in Figure 4 we show $10^{\circ}$ increments because it is easier to view). For the instrument description we import the parameters calibrated with the standard, and we only refine the error in centering (two parameters: $x, y$ ) along with the general intensity and the background. We can refine the unit cell parameter of the phase $(a)$ as both the detector distance and the wavelength are kept constant.

After refining instrument parameters, backgrounds and lattice parameter we proceed with the refinement of texture and microstructure. The strategy is similar to a normal Rietveld refinement, taking into account that to fit intensities correctly we also need to refine the texture.

There is only one phase in this sample and it is a facecentered cubic copper structure with some nickel as atomic substitution. We start from a pure copper phase and substitute part of the copper with nickel ( 25 at.\% of $\mathrm{Ni}$ ) and adjust the unit cell parameter to accommodate this change. For the microstructure, we choose the default line broadening method in MAUD that correspond to the simple "Delft" model (see De Keijser et al., 1982; Delhez et al., 1993) and isotropic crystallite size and microstrain for the phase. This simple model that attributes Lorentzian broadening for the finite crystallite size and Gaussian broadening for the microstrain is sufficient to describe the diffraction profile of the $\mathrm{Cu}-\mathrm{Ni}$ phase in this sample. No anisotropic peak broadening was observed.

For the texture model, we choose EWIMV (the algorithm is described in Lutterotti et al., 2004) that has all the features of the direct discrete WIMV method (Matthies and Vinel, 1982) but has been reformulated to work within the Rietveld method. The EWIMV is able to work with arbitrary pole-figure grids. The value $P_{h k l}$ on the pole figure for a certain reflection $h k l$ can be computed using a numerical integration (here denoted with an integral) of ODF values over the proper path inside the orientation space that is considered to be discrete (divided onto quasi-rectangular cells). Instead of using only the ODF values in the cells that the path is crossing, in EWIMV all neighboring cells are also included, weighing their contribution by the inverse of the distance of their center from the path $r$ (a "tube projection", Pawlik,
1986):

$$
P_{h}=\int \frac{1}{K} \sum_{i=1}^{N} \frac{f_{i}}{r_{i}}
$$

where

$$
K=\sum_{i=1}^{N} \frac{1}{r_{i}}
$$

$f_{i}$ is the ODF value in the cell $i$ and $N$ is the number of cells, including the neighboring ones. The use of such a tube projection ensures that the resulting orientation distribution will be sufficiently smooth, also in the case of poor pole-figure coverage. There are other texture models available in MAUD. The harmonic model can be useful for weak textures, especially of phases with high crystal symmetry, but it suffers from "ghost" effects because of omission of odd coefficients in the harmonic expansion. For very strong textures standard functions (fibers or spheres in the orientation space) can be used (this will be demonstrated in Part II for shale). Different texture models have been discussed by Kocks et al. (2000, Chapter 3).

It is important to ensure a sufficient coverage for a single diffraction image with no more than nine reflections over the entire $2 \theta$ range measured (including the partially visible reflections on the corners of the image). We start with a cell size of $15^{\circ}$ in EWIMV and no sample symmetry. At the end of the refinement with MAUD, we check the quality of the fit comparing measured and calculated model patterns graphically with a 2D display (Figure 6) and evaluating $R$ factor values. There are different $R$ factors to evaluate the quality of the refinement; some apply to the Rietveld refinement of diffraction data and others to the texture calculation from experimental pole density values. For both we use $R_{\mathrm{w}}$ that is the weighted $R$-factor (Table II). Note that the $R$-factor is a single number to describe a very complex situation (as discussed by Toby, 2006) and in the end the 2D display is more reliable to assess the quality of fit, including texture (Figure 6). The coverage of the orientation space by data can be assessed with a function that calculates the number of "hits" (number of integration paths through each cell in orientation space) and values of "weights" for each cell in

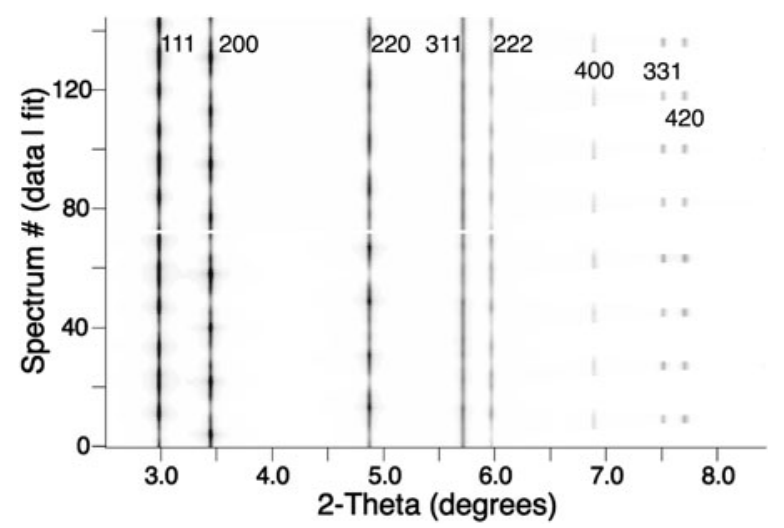

Figure 6. Nickel coin. Observed (bottom) and calculated 72 diffraction patterns (top) at end of refinement. $\omega=0^{\circ}$ image. "Plot2D" display. Lattice plane indices are indicated. 


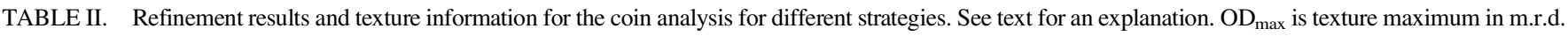

\begin{tabular}{|c|c|c|c|c|c|c|c|c|c|c|}
\hline \multicolumn{4}{|c|}{ Rietveld } & \multicolumn{3}{|c|}{ EWIMV } & \multicolumn{4}{|c|}{ OD coverage } \\
\hline Images & $R_{\mathrm{w}}(\%)$ & Parameters & Data number & Cell size & $R_{\mathrm{w}}(\%)$ & OD max & Weight min & Weight max & Hits min & Hits max \\
\hline 1 & 27.2 & 12 & 50799 & $15^{\circ}$ & 23.3 & 57.4 & 0.073 & 0.91 & 42 & 195 \\
\hline 1 & 17.3 & 12 & 50799 & $10^{\circ}$ & 10.9 & 50.0 & 0.014 & 0.34 & 14 & 99 \\
\hline 1 & 14.4 & 12 & 50799 & $7.5^{\circ}$ & 7.1 & 23.7 & 0.0009 & 0.19 & 3 & 75 \\
\hline 5 & 23.1 & 48 & 255155 & $10^{\circ}$ & 22.3 & 48.0 & 0.062 & 1.62 & 104 & 470 \\
\hline 5 & 20.3 & 48 & 255155 & $7.5^{\circ}$ & 18.8 & 35.4 & 0.013 & 0.81 & 43 & 296 \\
\hline 5 & 16.9 & 48 & 255155 & $5^{\circ}$ & 10.4 & 48.7 & 0.0015 & 0.31 & 10 & 156 \\
\hline
\end{tabular}

the orientation space (this plot can be activated from inside the EWIMV options panel after at least one computation is performed).
In the MAUD console, we can also retrieve the values for the $R$ factors of the texture computation and the minimummaximum values of "hits" and "weights" for the coverage.
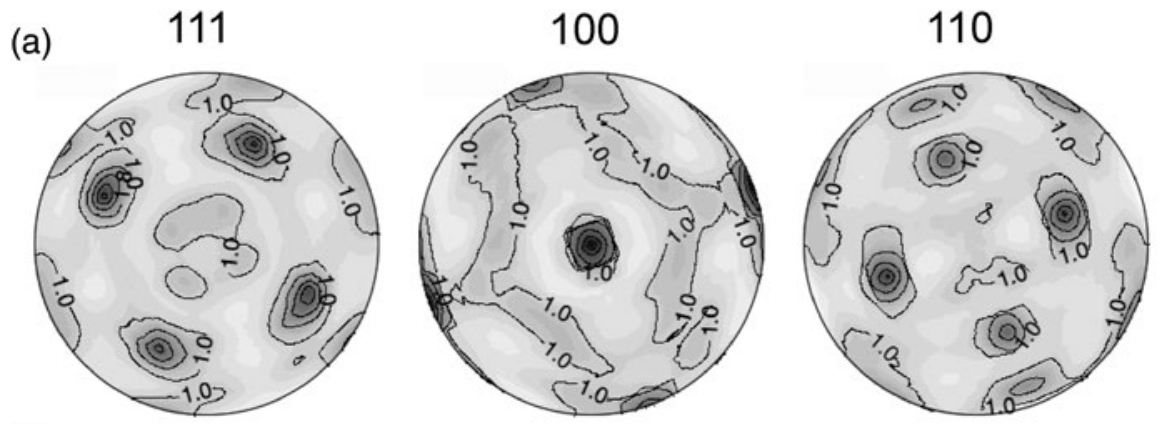

(b)
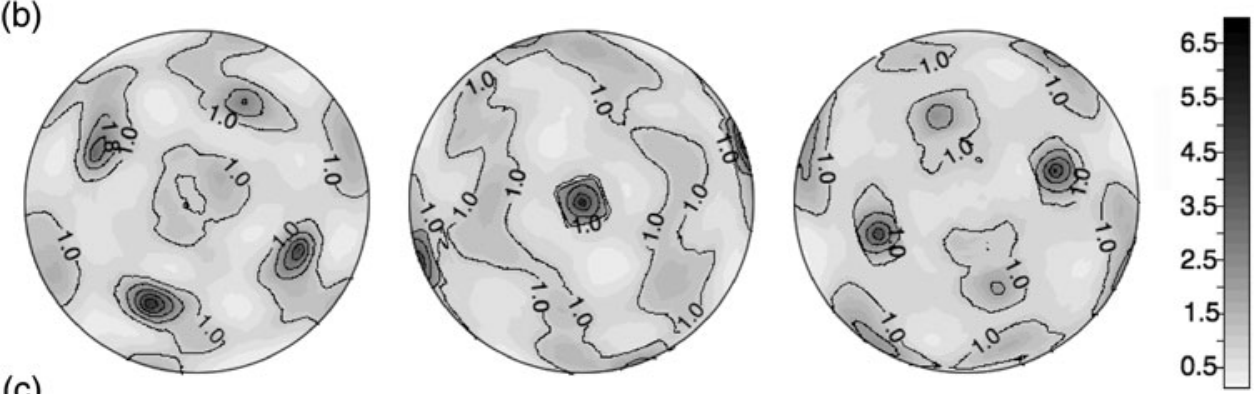

(c)
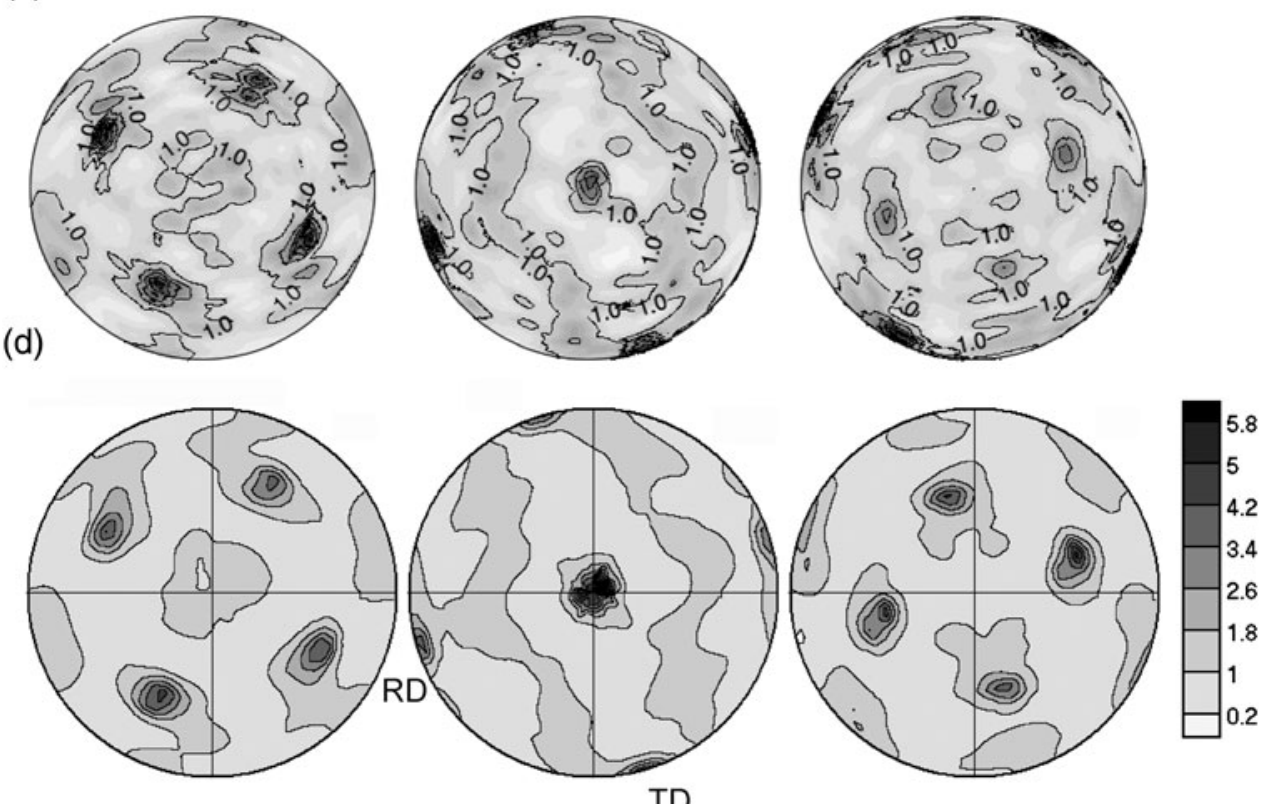

Figure 7. Plot of pole figures, comparing different coverages. (a) One image, $10^{\circ} \mathrm{OD}$ resolution; (b) five images, $10^{\circ} \mathrm{OD}$ resolution; (c) five images, $5^{\circ} \mathrm{OD}$ resolution; and (d) five images, $10^{\circ}$ OD resolution, processed with BEARTEX. Equal area projection, upper hemisphere, and pole densities in multiples of random distribution. 
Looking at Table II, using $15^{\circ}$ cells and only one image, the minimum number of hits is 42 and also the weight is not too low. But as we decrease the cell size the "hits" number decreases (at the same time $R_{w}$ decreases). For $7.5^{\circ}$ cells we have a minimum of three hits and less than $1 \mathrm{E}-3$ for the weight. Note that three hits is the limit for WIMV to resolve an orientation. By adding the other four images collected at different $\omega$ angles we see a pronounced increase in the coverage. Even in the case of $7.5^{\circ}$ cells we have the minimum of 43 "hits". The $R_{\mathrm{w}}$ increases as now we are using much more data and resolve more details of the ODF. Figure 7 compares pole figures for some models. The one image, $10^{\circ}$ cell case [Figure 7(a)] shows a remarkably similar pattern to the five image cases [Figure 7(b)]. The five images, $5^{\circ}$ cell case [Figure 7(c)] is considerably noisier and at the limit of resolution. After all, images were collected at $20^{\circ} \omega$ increments, resulting in very spotty $5^{\circ}$ ODF coverage. For the five image, $10^{\circ}$ cell case an ASCII file of the ODF was exported and further processed in BEARTEX (Wenk et al., 1998). Figure 7(d) compares the BEARTEX plot with a corresponding MAUD plot [Figure 7(b)]. From the microstructure model in MAUD we get a mean dimension of the coherently scattering crystallites around $500 \mathrm{~nm}$ and the true grain size may be even larger. Generally, for sizes larger than $200 \mathrm{~nm}$ diffraction patterns become almost insensitive to crystallite size.

The texture is a strong cube texture superposed on a weak rolling texture. In a cube texture, $\{100\}$ is parallel to rolling direction (RD in Figure 7(d)), transverse direction (TD) and the direction normal to the rolling plane (coin surface) in the center of the 100 pole figures. Cube is indicative of recrystallization (e.g., Schmidt and Lücke, 1979) and for a discussion of the transition from rolling to cube during recrystallization see Figure 40 in Chapter 4 of Kocks et al. (2000).

The full step-by-step procedure in MAUD to perform texture analysis on the coin sample is summarized in Appendix 2 (available online at http://www.journals.cambridge.org/PDJ) and corresponding data files can be downloaded from http://PD-journal.

\section{CONCLUSIONS}

Synchrotron X-rays provide a powerful method for quantitative texture analysis of materials. Depending on the sample size, beam size, and wavelength, small $\left(<100 \mu \mathrm{m}^{3}\right)$ to large volumes $\left(>200 \mathrm{~mm}^{3}\right)$ can be analyzed. Compared to neutron diffraction, electron backscatter diffraction or pole-figure goniometry, data acquisition is fast and images display immediately qualitative texture effects. 2D diffraction images are investigated with the modified Rietveld method, allowing for refining microstructural parameters, phase volume fractions and texture. For simple materials (in this case a coin), the refinement is straightforward and much can be done with an automatic wizard in the MAUD software within a few minutes.

\section{ACKNOWLEDGEMENTS}

This project was supported by the NSF (grant no. EAR-0836402) and DOE (grant no. DE-FG02-05ER15637). We greatly appreciate access to beamline 11-ID-C at APS of Argonne National Laboratory and help from Chris Benmore and Yang Ren. The tutorial was developed as part of a workshop at the Advanced Light Source in fall 2012, supported by CDAC, APS (BESSRC), NSF-COMPRES, ALS, and STONE-LANL.
We would like to thank Juan Gomez-Barreiro, Pam Kaercher and Waruntorn Kanitpanyacharoen for useful comments and valuable input from the editor B. Toby and three reviewers that helped us improve the manuscript.

\section{SUPPLEMENTARY MATERIALS AND METHODS}

The supplementary material for this article can be found at http://www.journals.cambridge.org/PDJ.

Battocchio, F., Monteiro, P. J. M., and Wenk, H.-R. (2012). "Rietveld refinement of the structures of $1.0 \mathrm{C}-\mathrm{S}-\mathrm{H}$ and $1.5 \mathrm{C}-\mathrm{S}-\mathrm{H}$, , Cem. Concr. Res. 42, 1534-1548.

Chantler, C. T., Tran, C. Q., and Cookson, D. J. (2004). "Precise measurement of the lattice spacing of $\mathrm{LaB}_{6}$ standard powder by the $\mathrm{x}$-ray extended range technique using synchrotron radiation," Phys. Rev. A 69, 1-11.

De Keijser, Th. H., Langford, J. I., Mittemeijer, E. J., and Vogels, A. B. P. (1982). "Use of the Voigt function in a single-line method for the analysis of X-ray diffraction line broadening," J. Appl. Crystallogr. 15, 308-314.

Delhez, R., de Keijser, Th. H., Langford, J. I., Louër, D., Mittemeijer, E. J., and Sonneveld, E. J. (1993). "Crystal imperfection broadening and peak shape in the Rietveld method" in "The Rietveld Method" edited by R. A. Young (Oxford University Press, Oxford, UK), pp. 132-166.

Grässlin, J., McCusker, L. B., Baerlocher, C., Gozzo, F., Schmitt, B., and Lutterotti, L. (2013). "Advances in exploiting preferred orientation in the structure analysis of polycrystalline materials," J. Appl. Crystallogr. 46, 173-180.

Hammersley, A. P. (1998). Fit2D: V99.129 Reference Manual Version 3.1. Internal Report ESRF - 98 - HA01.

Kocks, U. F., Tomé, C. N., and Wenk, H-R. (2000). Texture and Anisotropy: Preferred Orientations in Polycrystals and their Effect on Materials Properties (Cambridge University Press, Cambridge), 2nd ed., p. 677.

Larson, A. C. and Von Dreele, R. B. (2004). General Structure Analysis System (GSAS) (Report LAUR 86-748). Los Alamos, New Mexico: Los Alamos National Laboratory.

Le Bail, A., Duroy, H., and Fourquet, J. L. (1988). "A $b$-initio structure determination of $\mathrm{LiSbWO}_{6}$ by X-ray powder diffraction,” Mater. Res. Bull. 23, 447-452.

Lonardelli, I., Wenk, H.-R., Lutterotti, L., and Goodwin, M. (2005). "Texture analysis from synchrotron diffraction images with the Rietveld method: dinosaur tendon and salmon scale," J. Synchrotr. Radiat. 12, 354-360.

Lutterotti, L. (2010). "Total pattern fitting for the combined size-strainstress-texture determination in thin film diffraction," Nucl. Instrum. Methods Phys. Res. B 268, 334-340.

Lutterotti, L. and Bortolotti, M. (2005). "Algorithms for solving crystal structure using texture," Acta Crystallogr. A61, C158-C159.

Lutterotti, L., Matthies, S., Wenk, H.-R., Schultz, A. S., and Richardson, J. W. (1997). "Combined texture and structure analysis of deformed limestone from time-of-flight neutron diffraction spectra," J. Appl. Phys. 81, 594-600.

Lutterotti, L., Chateigner, D., Ferrari, S., and Ricote, J. (2004). "Texture, residual stress and structural analysis of thin films using a combined X-ray analysis," Thin Solid Films 450, 34-41.

Matthies, S. and Vinel, G. W. (1982). "On the reproduction of the orientation distribution function of texturized samples from reduced pole figures using the conception of a conditional ghost correction," Phys. Status Solidi b 112, K111-K114.

Pawlik, K. (1986). "Determination of the orientation distribution function from pole figures in arbitrarily defined cells," Phys. Status Solidi b 134, 477-483.

Rietveld, H. M. (1969). "A profile refinement method for nuclear and magnetic structures,” J. Appl. Crystallogr. 2, 65-71.

Schmidt, U. and Lücke, K. (1979). "Recrystallization textures of silver, copper and $\alpha$-brasses with different zinc-contents as a function of the rolling temperature," Texture Cryst. Solids 3, 85-112.

SRM 660b (2010). Lanthanum Hexaboride Powder Line Position and Line Shape Standard for Powder Diffraction (National Institute of Standards and Technology; US Department of Commerce, Gaithersburg, MD), pp. 1-5. 
SRM 674b (2012). Cerium Oxide (Quantitative Powder Diffraction Standard) (National Institute of Standards and Technology; US Department of Commerce, Gaithersburg, MD), pp. 1-5.

Toby, B. H. (2006). " $R$ factors in Rietveld analysis: how good is good enough?" Powder Diffr. 21, 67-70.

Vogel, S. C., Hartig, C., Lutterotti, L., Von Dreele, R. B., Wenk, H.-R., and Williams, D. J. (2004). "Texture measurements using the new neutron diffractometer HIPPO and their analysis using the Rietveld method." Powder Diffr. 19, 65-68.

Von Dreele, R. B. (1997). "Quantitative texture analysis by Rietveld refinement," J. Appl. Crystallogr. 30, 517-525.

Wenk, H.-R., Matthies, S., Donovan, J., and Chateigner, D. (1998). "BEARTEX: a windows-based program system for quantitative texture analysis,” J. Appl. Crystallogr. 31, 262-269.
Wenk, H.-R., Lutterotti, L., and Vogel, S. C. (2010). "Rietveld texture analysis from TOF neutron diffraction data," Powder Diffr. 25, 283-296.

Wenk, H.-R., Vasin, R. N., Kern, H., Matthies, S., Vogel, S. C., and Ivankina, T. I. (2012). "Revisiting elastic anisotropy of biotite gneiss from the Outokumpu scientific drill hole based on new texture measurements and texture-based velocity calculations," Tectonophysics 570-571, 123-134.

Wenk, H.-R., Kaercher, P., Kanitpanyacharoen, W., Lutterotti, L., Miyagi, L., and Vasin, R. N. (2014). "Rietveld texture analysis from synchrotron diffraction images. II. Complex multiphase materials and diamond anvil cell experiments," Powder Diffr. (in press).

Yashima, M., Ishimura, D., Yamaguchi, Y., Ohoyama, K., and Kawachi, K. (2003). "High-temperature neutron powder diffraction study of cerium dioxide $\mathrm{CeO}_{2}$ up to $1770 \mathrm{~K}$," Chem. Phys. Lett. 372, 784-787. 